\title{
Monodisperse Liquid Crystal Network Particles Synthesized via Precipitation Polymerization
}

\author{
Xiaohong Liu, ${ }^{\dagger}\|\|$ Yifei Xu, ${ }^{\ddagger}, \|$ Johan P.A. Heuts, ${ }^{\S, \| \odot ~ M i c h a e l ~ G . ~ D e b i j e, ~}{ }^{\dagger}$ \\ and Albert P.H.J. Schenning $*, \dagger, \|(0)$
}

\begin{abstract}
${ }^{\dagger}$ Stimuli-Responsive Functional Materials and Devices, ${ }^{\dagger}$ Laboratory of Materials and Interface Chemistry and Center for Multiscale Electron Microscopy, and ${ }^{\S}$ Supramolecular Polymer Chemistry group, Department of Chemical Engineering and Chemistry, Eindhoven University of Technology, $5600 \mathrm{MB}$ Eindhoven, The Netherlands

"Institute for Complex Molecular Systems, Eindhoven University of Technology, P.O. Box 513, 5600 MB, Eindhoven, The Netherlands
\end{abstract}

\section{Supporting Information}

\begin{abstract}
The production of liquid crystalline (LC) polymer particles with a narrow size distribution on a large scale remains a challenge. Here, we report the preparation of monodisperse, cross-linked liquid crystalline particles via precipitation polymerization. This versatile and scalable method yields polymer particles with a smectic liquid crystal order. Although the LC monomers are randomly dissolved in solution, the oligomers self-align and LC order is induced. For the polymerization, a smectic LC monomer mixture consisting of cross-linkers and benzoic acid hydrogen-bonded dimers is used. The average diameter of the particles increases at higher

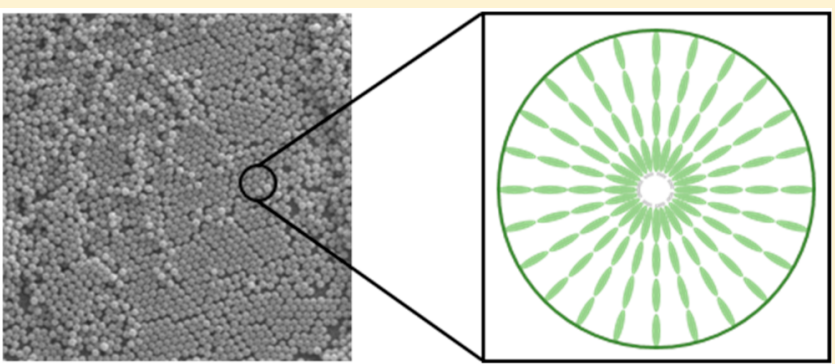
polymerization temperatures and in better solvents, whereas the monomer and initiator concentration have only minor impact on the particle size. After deprotonating of the benzoic acid groups, the particles show rapid absorption of a common cationic dye, methylene blue. The methylene blue in the particles can be subsequently released with the addition of $\mathrm{Ca}^{2+}$, while monovalent ions fail to trigger the release. These results reveal that precipitation polymerization is an attractive method to prepare functional LC polymer particles of a narrow size distribution and on a large scale.
\end{abstract}

\section{INTRODUCTION}

Liquid crystalline (LC) polymer particles exhibit unique functional properties due to their well-defined ordered structure. Anisotropic order LC particles can be manipulated with optical tweezers ${ }^{1}$ or rotated with electric fields, ${ }^{2}$ while chiral nematic ordered LC particles are able to reflect light ${ }^{3,4}$ or can be used as microlasers. ${ }^{5}$ Smectic LC particles have been reported that show superior properties as absorbents, as fast absorption and desorption of the dye and high absorption capacity were achieved. ${ }^{6}$

Several techniques have been used to prepare LC polymer particles, including miniemulsion, suspension, microfluidic, and dispersion polymerizations. Small LC particles $(\sim 200 \mathrm{~nm}$ in diameter) have been prepared via miniemulsion polymerization, ${ }^{7-9}$ while larger particles were prepared by suspension polymerization. ${ }^{3,4,6,10}$ In both cases, however, the particle size distributions were broad. Polydisperse particles show nonuniform behavior as particles of different diameter have different functional properties. For applications, such as photonic crystals or drug delivery monodisperse polymer particles are desired.

By using microfluidics, monodisperse particles between 200 and $500 \mu \mathrm{m}$ have been fabricated. ${ }^{1-15}$ However, microfluidic production faces difficulties in scaling-up. LC polymer particles prepared by dispersion polymerization ${ }^{1,2,16,17}$ had a low dispersity, and the technique is scalable. Previous studies have shown that introduction of cross-linkers and functional groups tends to destabilize the dispersion and lead to coagulum, poor morphology, and a high polydispersity. ${ }^{18,19}$ This drawback severely limits the applicability of dispersion polymerization.

Precipitation polymerization is an attractive method to prepare polymer particles with narrow size distributions on a relatively large scale. In precipitation polymerization, radicals first grow in the solution until the solubility limit is reached, and the oligomers precipitate and form preliminary particles, which further grow primarily by capturing propagating radicals from the solution. ${ }^{20}$ Monodisperse, highly cross-linked, and functional polymer particles have been prepared by this method. ${ }^{21,22}$ The polymerization method does not require specific equipment or surfactants. Moreover, particles with complex structures can also be prepared, including core-shell and hollow particles. ${ }^{23-27}$ To date, however, precipitation

Received: September 4, 2019

Revised: October 18, 2019

Published: October 29, 2019 
Table 1. Polymerization Condition at Each Run and the Corresponding Results

\begin{tabular}{|c|c|c|c|c|c|c|c|c|}
\hline entry & {$[\text { initiator }]^{a}$} & {$[\text { monomer }]^{b}$} & solvent & temp $\left({ }^{\circ} \mathrm{C}\right)$ & $D_{\mathrm{n}}(\mu \mathrm{m})$ & $C_{\mathrm{V}}(\%)$ & $D_{z}(\mu \mathrm{m})$ & yield (\%) \\
\hline 1 & $1 \%$ & $2 \%$ & phenyl acetate & 95 & 0.62 & 7.5 & 0.79 & 12 \\
\hline 2 & $2 \%$ & $2 \%$ & phenyl acetate & 95 & 1.05 & 6.5 & 1.13 & 75 \\
\hline 3 & $3 \%$ & $2 \%$ & phenyl acetate & 95 & 0.92 & 5.0 & 1.06 & 71 \\
\hline 4 & $4 \%$ & $2 \%$ & phenyl acetate & 95 & 0.97 & 3.7 & 1.08 & 73 \\
\hline 5 & $2 \%$ & $1 \%$ & phenyl acetate & 95 & 0.82 & 5.4 & 0.84 & 31 \\
\hline 6 & $2 \%$ & $3 \%$ & phenyl acetate & 95 & 0.95 & 6.7 & 1.02 & 67 \\
\hline 7 & $2 \%$ & $4 \%$ & phenyl acetate & 95 & 1.03 & 6.8 & 1.07 & 70 \\
\hline 8 & $2 \%$ & $2 \%$ & phenyl acetate & 65 & 0.70 & 5.2 & 0.88 & 89 \\
\hline 9 & $2 \%$ & $2 \%$ & ethyl acetate & 65 & 1.44 & 5.4 & 1.79 & 57 \\
\hline
\end{tabular}

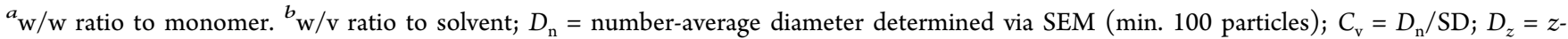
average diameter determined via DLS; yield = weight of particles/weight of monomers.

(a)

)
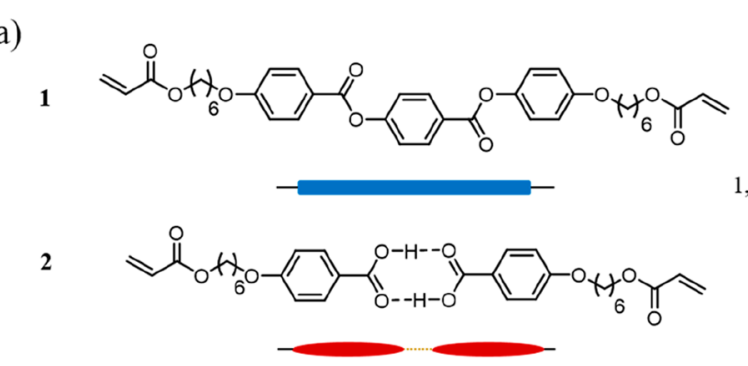

1,1-Bis(tert-butylperoxy)cyclohexane (Luperox 331 P80)

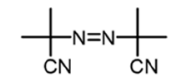

Azobisisobutyronitrile (AIBN)

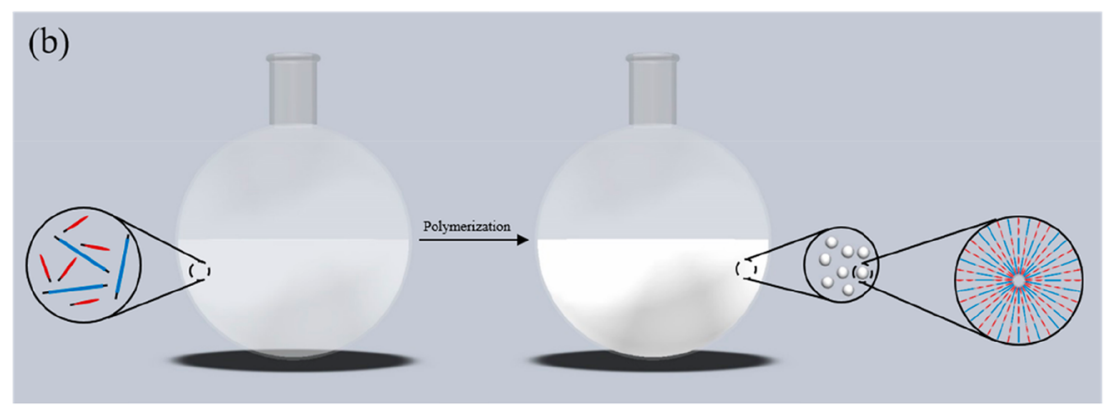

Figure 1. (a) Chemical structure of the monomers and initiators. (b) Schematic representation of precipitation polymerization method to synthesize liquid crystalline polymer particles.

polymerization has never been used to prepare LC polymer particles.

Previously, we reported the suspension polymerization of nanoporous polymer particles based on hydrogen-bonded smectic LC benzoic acid monomers. ${ }^{6}$ After polymerization, polydisperse particles with an average diameter of $1.4 \mu \mathrm{m}$ were obtained in a low yield. Treatment with base resulted in nanoporous polymer particles with an onionlike order. Dye absorption experiments demonstrated a high absorption capacity and fast absorption kinetics.

We now describe the preparation of nanoporous LC particles by precipitation polymerization. This versatile and scalable method yields polymer particles with a low dispersity of diameters between 0.7 and $1.4 \mu \mathrm{m}$. Although the LC monomers are randomly dissolved in solution, well-defined particles with a smectic LC order are obtained. The average diameter of the particles increases at higher polymerization temperatures and in better solvents, whereas the monomer and initiator concentration have only a minor impact on the particle size. The particles can be used to rapidly absorb and selectively release molecules in the presence of divalent cations. Hence precipitation polymerization is an attractive new method to prepare functional liquid crystalline polymer particles of a narrow size distribution on a large scale.

\section{EXPERIMENTAL SECTION}

2.1. Materials. 4-(6-Acryloxyhexyl-1-oxy)benzoic acid (1) and 4((4-(6-(acryloyloxy)hexyloxy)phenoxy) carbonyl)phenyl 4-(6(acryloyloxy)hexyloxy) benzoate (2) were purchased from Synthon Chemicals, Germany. Phenyl acetate $(>98 \%)$ was purchased from TCI Europe. Other solvents were purchased from Biosolve. Luperox 331 P80 (1,1-bis(tert-butylperoxy)cyclohexane, $80 \mathrm{wt} \%$ in benzyl butyl phthalate, technical grade), 2,2'-azobis(2-methylpropionitrile) (AIBN, 98\%), potassium hydroxide ( $\mathrm{KOH}, 85 \%)$, calcium chloride $\left(\mathrm{CaCl}_{2}\right)$, lithium chloride $(\mathrm{LiCl})$, potassium chloride $(\mathrm{KCl})$, and sodium chloride $(\mathrm{NaCl})$ were purchased from Sigma-Aldrich. Methylene blue (MB) was purchased from Acros Organics.

2.2. Experimental Procedures. 2.2.1. Synthesis of the Liquid Crystalline Particles. First, $50 \mathrm{mg}$ of $\mathbf{1}$ and $50 \mathrm{mg}$ of $\mathbf{2}$ were added to a $50 \mathrm{~mL}$ round-bottom flask; the flask was then pumped and backfilled with argon 3 times. The initiator (AIBN for polymerization at $65^{\circ} \mathrm{C}$, Luperox $331 \mathrm{P} 80$ for polymerization at $95^{\circ} \mathrm{C}$ ) was dissolved in ethyl acetate or phenyl acetate and added to the flask (see Table 1 for details). The flask was put into an oil bath preheated to the polymerization temperature for $24 \mathrm{~h}$. After polymerization, the suspension was centrifuged and washed with ethanol to obtain the particles. 

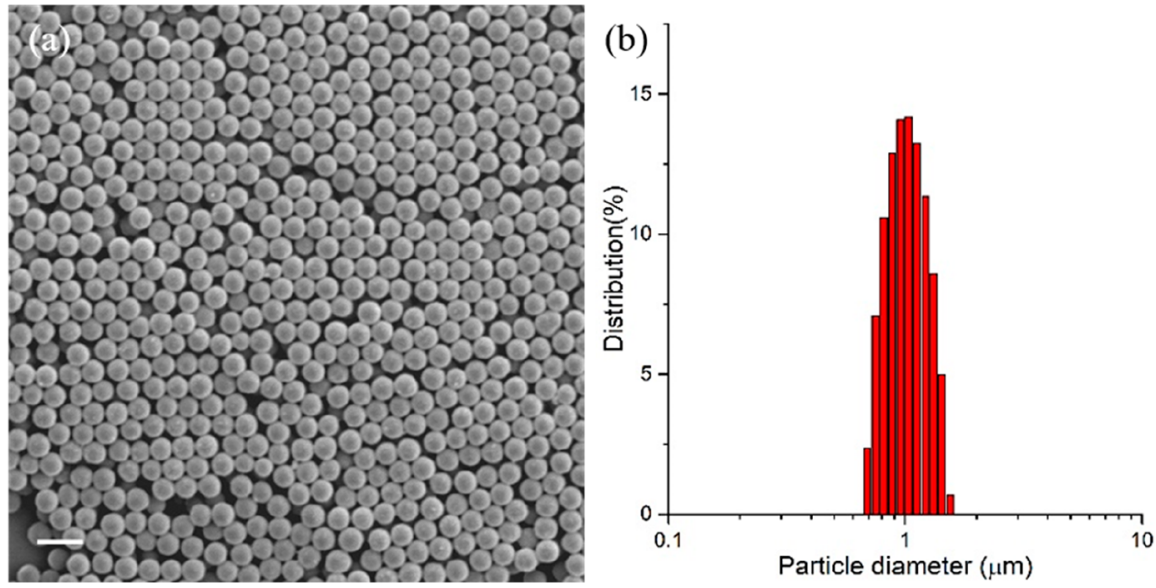

Figure 2. SEM image (a) and DLS (b) of particles synthesized in entry 4 . Scale bar $=2 \mu \mathrm{m}$.

2.2.2. Fabrication of Nanoporous Particles. The pristine particles were sonicated in $10 \mathrm{mM} \mathrm{KOH}$ solution at room temperature until completely dispersed. Then the particles were centrifuged and washed with deionized water twice.

2.3. Absorption and Release of Methylene Blue. For the absorption kinetics, $10 \mu \mathrm{L}$ of $\mathrm{MB}$ solution $\left(\mathrm{MB} / \mathrm{COO}^{-}=1\right)$ was added to $2.95 \mathrm{~mL}$ of $0.01 \mathrm{M} \mathrm{KOH}$ solution in a $3 \mathrm{~mL}$ cuvette, and the absorption of the solution was measured. Then $36.7 \mu \mathrm{L}$ of $0.5 \mathrm{mg} / \mathrm{mL}$ particle suspension was added to the solution, and the absorption spectrum of the solution was measured every minute.

To monitor the release kinetics, $10 \mu \mathrm{L}$ of $\mathrm{MB}$ solution and $36.7 \mu \mathrm{L}$ of $0.5 \mathrm{mg} / \mathrm{mL}$ particle suspension $\left(\mathrm{MB} / \mathrm{COO}^{-}=1\right)$ were added to $2.95 \mathrm{~mL}$ of $0.01 \mathrm{M} \mathrm{KOH}$ solution and equilibrated for an hour. Then $2.7 \mathrm{~mL}$ of the solution was transferred to a $3 \mathrm{~mL}$ cuvette, and the absorption of the solution was measured. Then $300 \mu \mathrm{L}$ of $10 \mathrm{mM}$ salt solution was added, and the absorption spectrum of the solution was measured every minute to monitor the release of $\mathrm{MB}$.

2.4. Characterization. Polarized optical microscopy (POM) images were taken with a Leica CTR6000 polarized optical microscope and a Leica DFC 420C camera. The temperature was controlled with a Linkam temperature control stage. Differential scanning calorimetry (DSC) curves were measured with a DSC Q2000 from TA Instruments, with $3{ }^{\circ} \mathrm{C} / \mathrm{min}$ temperature ramp from -20 to $130{ }^{\circ} \mathrm{C}$ and $5 \mathrm{~min}$ isotherm. The second heating and cooling cycles were used for characterization. Attenuated total reflection Fourier transfer infrared spectra (ATR-IR) were measured with a Varian 670-IR spectrometer. X-ray diffraction (XRD) was performed on a Ganesha lab instrument with a GeniX-Cu ultralow divergence source producing X-ray photons with a wavelength of $1.54 \AA$ and a flux of $1 \times 10^{8} \mathrm{ph} / \mathrm{s}$. Scattering patterns were collected with a Pilatus $300 \mathrm{~K}$ silicon pixel detector with $478 \times 619$ pixels, each $172 \mu \mathrm{m}^{2}$ in size. Dynamic light scattering (DLS) was measured with Anton Paar Litesizer 500 in $1 \mathrm{mM} \mathrm{KCl}$ solution in backscattering mode at $25^{\circ} \mathrm{C}$. UV-vis spectra were measured with a Shimadzu UV-3102 PC. Scanning electron microscopy (SEM) images were taken with a JEOL TM $220 \mathrm{~A}$. The average size and coefficient of variation of the particle size distribution $\left(C_{\mathrm{v}}=\frac{\bar{d}}{\mathrm{SD}}\right)$ were measured and calculated over 100 particles per sample.

\section{RESULTS AND DISCUSSION}

3.1. LC Monomer Mixture. The LC monomer mixture consists of a hydrogen-bonded benzoic acid monomer (1) that attracts cations when deprotonated and an LC cross-linker (2) to maintain the network, in a $1 / 1 \mathrm{w} / \mathrm{w}$ ratio (Figure $1 \mathrm{a}$ ). The phase behavior of the mixtures was studied via POM and DSC. An isotropic-nematic transition was observed at around 115 ${ }^{\circ} \mathrm{C}$, and the mixture further converts into a smectic A phase at around $105^{\circ} \mathrm{C}$; this phase is maintained at room temperature (Figure S1).

3.2. Synthesis of the Liquid Crystalline Particles by Precipitation Polymerization. The LC particles were synthesized by precipitation polymerization. The solvent is a vital factor for precipitation polymerization, as it needs to dissolve both the monomers and free radical initiator but also allow oligomers reaching a critical chain length to precipitate and form the preliminary particles. ${ }^{20}$ Upon screening of multiple solvents (including acetonitrile, toluene, and isopropyl alcohol, among others), phenyl acetate and ethyl acetate were found to produce monodisperse particles. Monomers, initiator, and solvent were added to an oxygen-free flask and polymerized at different temperatures for $24 \mathrm{~h}$ to yield the particles. Fourier transform infrared spectroscopy (FTIR) confirmed the conversion of the acrylate moieties (Figure S4). We first investigated the effect of monomer concentration, initiator concentration, solvent, and temperature on the average particle size and size distribution (Table 1 and Figure S3). The particle size distribution was analyzed by SEM and DLS, and a typical example (entry 4 in Table 1) is given in Figure 2. SEM showed spherical objects with a diameter of $0.97 \mu \mathrm{m}$. The coefficient of variation $\left(C_{\mathrm{v}}\right)$, which is a measure of dispersity, was $3.7 \%$, meaning the particles are monodisperse. Only one narrow peak was found in DLS, and the $z$ average diameter was determined as $1.08 \mu \mathrm{m}$.

The effect of initiator and monomer concentrations on the final particle size and distribution was performed in phenyl acetate at $95{ }^{\circ} \mathrm{C}$ using Luperox $331 \mathrm{P} 80$ as initiator. Initiator and monomer concentrations were varied from $1 \%$ to $4 \%$ (entries 1-7). With increasing initiator and decreasing monomer concentrations, the coefficient of variation decreases slightly. The diameter of the particles was around $1 \mu \mathrm{m}$ regardless of the initiator and monomer concentrations if they were both $\geq 2 \%$. However, smaller particles and lower yields were observed when either the initiator or monomer concentration was low $(<2 \%)$, probably because the initiator efficiency was decreased, the oligomer chains had difficulty in forming preliminary particles, and the conversion of the monomers was limited under dilute conditions, also indicated by the low yield. When the polymerization was carried out at $65{ }^{\circ} \mathrm{C}$ and with AIBN as initiator (entry 8), smaller particles were obtained compared to polymerization performed at 95 ${ }^{\circ} \mathrm{C}$. The decreased particle size can be attributed to the decreased solubility of the oligomer chains at lower temper- 

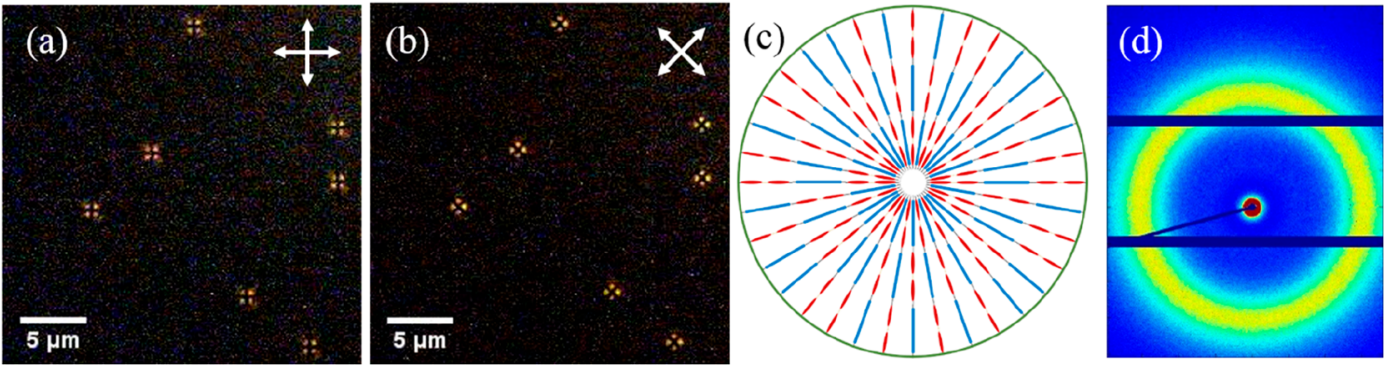

Figure 3. (a, b) POM images of the particles with cross polarizers. (c) Radial alignment of the liquid crystal molecules in the particles. (d) XRD of the particles. The particles used were entry 4. See also Figure 2 for SEM images and DLS result of the particles.
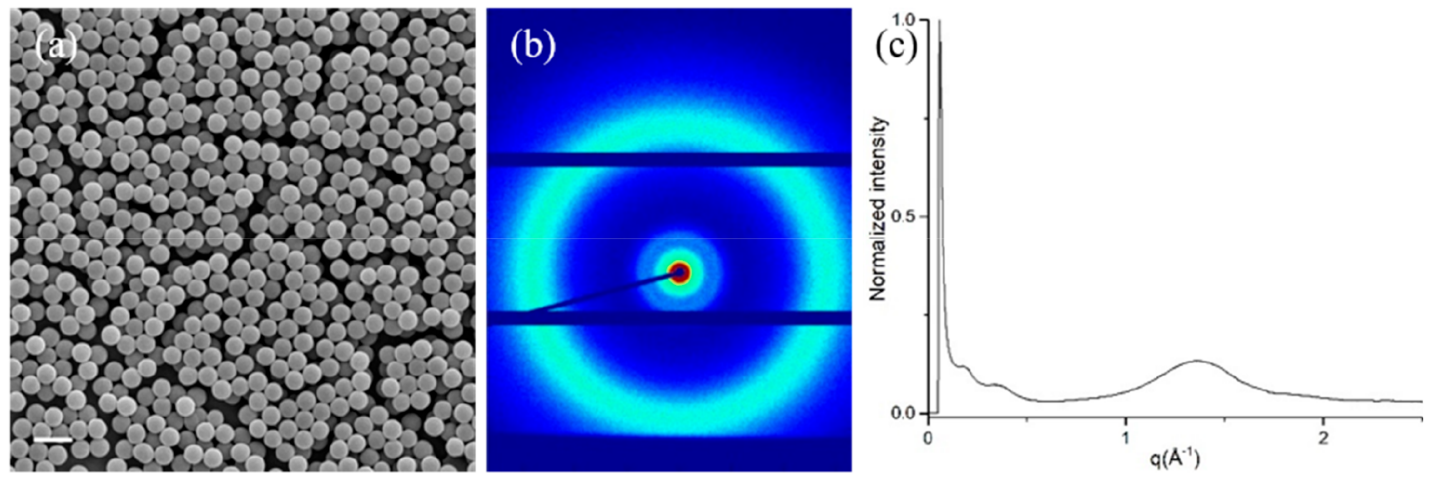

Figure 4. $(\mathrm{a}-\mathrm{c}) \mathrm{SEM}, \mathrm{XRD}$, and XRD 1D profile of the polymer particles after KOH treatment. Scale bar $=2 \mu \mathrm{m}$.

atures, which meant the nucleation occurs faster, resulting in the formation of smaller particles. Particles synthesized in ethyl acetate were larger than particles synthesized in phenyl acetate (entry 8 versus entry 9), as ethyl acetate is a better solvent for the polymers, resulting in slower nucleation and larger particles. As suggested in the literature, solvent and temperature affect the size and morphology of the particles through changing the Flory parameter, $\chi{ }^{28}$ With low $\chi$, the interaction between solvent and polymer is favorable and leads to an extended growth of oligomer chains in the solution and, as a result, gives larger particles.

Particles prepared in entry 4 were characterized in more detail and used in adsorption and release studies.

3.3. Characterization of the Particles. POM analysis using cross polarizers was used to investigate the internal structure and molecular alignment in the polymer particles. The particles were first suspended in water and then dried between two glass slides to be nicely dispersed and to eliminate Brownian motion. As shown in Figure $3 a$ and $3 b$, so-called Maltese crosses were observed in the same direction as the crossed polarizers. This indicates that the LC molecules align homeotropically, that is, perpendicular to the particle surface (Figure 3c). ${ }^{6}$ Interestingly, although the LC monomers are randomly dissolved, the oligomers apparently self-align inducing liquid crystalline order. The polymer particles were further characterized by XRD, revealing a peak at $0.4 \mathrm{~nm}$, which can be attributed to the intermolecular spacing of the molecules (Figure 3d). However, no layer spacing was observed which might be due to the low contrast in the particles (vide infra).

3.4. Formation of Nanoporous Particles. To show that the LC particles can be used to absorb and release molecules, nanoporous particles were prepared. The particles were treated with $0.01 \mathrm{M} \mathrm{KOH}$ solution to break the hydrogen bonding between $\mathrm{COOH}$ groups and transform them into $\mathrm{COO}^{-} \mathrm{K}^{+}$ salts. IR spectra confirmed complete deprotonation, as the peaks corresponding to hydrogen bonds at 2500-2700 and $1677 \mathrm{~cm}^{-1}$ disappeared completely, and the peaks corresponding to asymmetric and symmetric stretching of carboxylate salt at 1545 and $1392 \mathrm{~cm}^{-1}$ appeared (Figure S4). The average size and size distribution of the particles remained unchanged during the $\mathrm{KOH}$ treatment (Figure 4a), most likely due to the high cross-linking density that prevented the polymer particles from swelling. A new signal at $3.4 \mathrm{~nm}$ and the corresponding second-order peak at $1.7 \mathrm{~nm}$ were observed in XRD, which were assigned to the layer spacing in the LC particles (Figure $4 \mathrm{~b}$ and $4 \mathrm{c}$ ). This layer spacing might be originating from the thermotropic smectic LC mixture used for the precipitation polymerization. The appearance of the layer spacing signal is probably due to the introduction of potassium into the particles. The "pristine" particles consist of only carbon, oxygen, and hydrogen, elements with low electron density, resulting in low contrast in XRD measurements. After hydrogen in the benzoic acid groups is replaced with potassium with higher electron density, the contrast is improved and the layer spacing becomes visible.

3.5. Absorption and Release Characteristics. The loading and release properties of the particles were investigated by using methylene blue as a probe. To an aqueous solution of $\mathrm{MB}$, nanoporous particles were added, and the absorption process was monitored by UV-vis measurement. The spectra of $\mathrm{MB}$ in solution and in particles were significantly different (shown in Figure $5 b$, inset). The absorption of $\mathrm{MB}$ results in a decrease in the absorbance at $665 \mathrm{~nm}$, which is most likely due to $\mathrm{MB}$ aggregation within the particles, as the local concentration is high. ${ }^{29-31}$ By plotting the absorbance at 665 $\mathrm{nm}$ versus time, the absorption was found to be completed within a few minutes, indicating fast absorption kinetics. The 
(a)
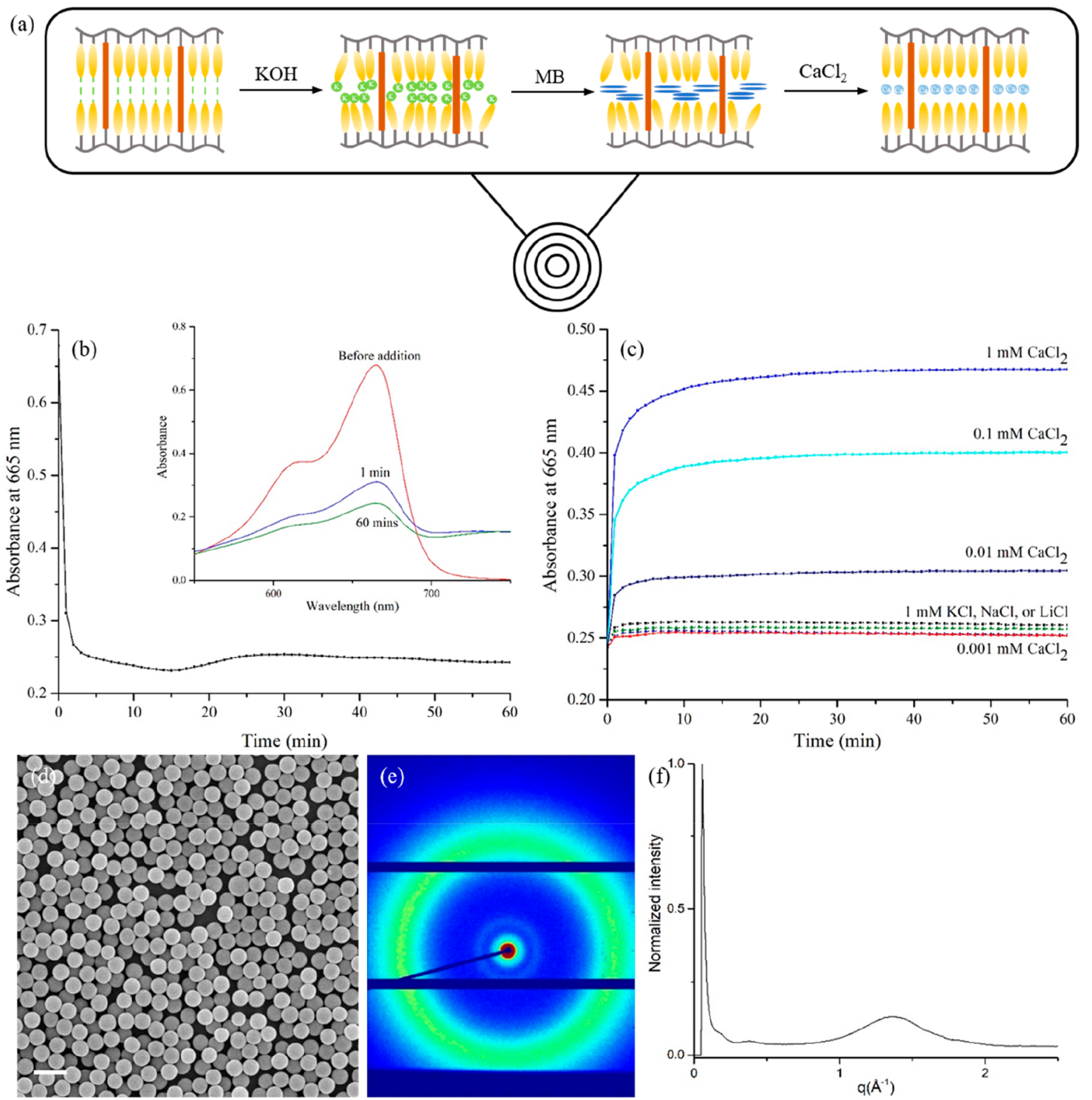

Figure 5. (a) Schematic representation of the MB absorption and release by the nanoporous polymer particles. (b) UV-vis measurement of the absorption process. UV-vis spectra of $M B$ in solution and MB absorbed by the particles are shown in the inset. (c) Time-resolved UV-vis measurement of the release process monitoring the peak absorbance of the MB. (d-f) SEM, XRD, and XRD 1D profile of the polymer particles containing $\mathrm{MB}\left(\mathrm{MB} / \mathrm{COO}^{-}=1\right)$. Scale bar $=2 \mu \mathrm{m}$.

MB-loaded particles were collected for SEM and XRD analysis. The particle diameter increased slightly to $1 \mu \mathrm{m}$ (Figure $5 \mathrm{~d}$ ), while the layer spacing remained as $3.4 \mathrm{~nm}$, although the intensity of peaks in XRD was lower, a result of the lower electron density and decreased contrast of MB (Figure 5e, f).

Different chloride salt solutions were added to the loaded particles to study the release of MB. Monovalent salts, including sodium chloride, lithium chloride, and potassium chloride, did not trigger the release of $\mathrm{MB}$, as the absorbance at $665 \mathrm{~nm}$ only changed slightly. However, when the bivalent salt calcium chloride was added, the absorbance increased, indicating that $\mathrm{MB}$ was released from the particles. The release kinetics were monitored in the same way as for the absorption process, and $\mathrm{MB}$ release was found to be completed within a few minutes, increasing with higher calcium chloride concentration. The minimum concentration required to trigger $\mathrm{MB}$ release was $0.01 \mathrm{mM}$ (Figure 5). The concentration of $\mathrm{Ca}^{2+}$ needed to release $\mathrm{MB}$ was much lower, and the release was faster than reported earlier, ${ }^{32,33}$ which might be the result of the high affinity of the well-defined porous structure within the particles.

\section{CONCLUSIONS}

We report the preparation of monodisperse, micrometer-size, highly cross-linked, and functionalized LC particles by versatile and scalable precipitation polymerization. Although the LC monomers are randomly dissolved in solution, the oligomers self-align, and liquid crystalline order is induced. The LC molecules align radially within the particles. The size of the particles can be tuned by both temperature and solvent, while 
the monomer and initiator concentration (>2\%) have only minor impacts. The particles could be used to absorb and release molecules in water which makes them attractive for water purification, drug delivery, and selective recovery of valuable chemicals. These results reveal that precipitation polymerization is an attractive method to prepare functional liquid crystalline polymer particles of a narrow size distribution and on a large scale. Preliminary experiments show that our precipitation polymerization method can also be applied to other LC mixtures to prepare monodisperse well-defined LC polymers particles (Figure S5).

\section{ASSOCIATED CONTENT}

\section{S Supporting Information}

The Supporting Information is available free of charge on the ACS Publications website at DOI: 10.1021/acs.macromol.9b01852.

Optical image of the monomer LC mixture under POM; DSC curve of the monomer LC mixture; SEM images of LC polymer particles synthesized in runs 1- 9; IR spectra of the monomer LC mixture, pristine polymer particles, and particles after $\mathrm{KOH}$ treatment; chemical structure of a nematic mixture; POM and SEM image of particles synthesized from the nematic mixture; particles synthesized with the monomer mixture mentioned in the maintext in a $9 / 1 \mathrm{w} / \mathrm{w}$ ratio $(\mathrm{PDF})$

\section{AUTHOR INFORMATION}

\section{Corresponding Author}

*E-mail address: A.P.H.J.Schenning@tue.nl.

\section{ORCID}

Johan P.A. Heuts: 0000-0002-9505-8242

Michael G. Debije: 0000-0001-8844-1115

Albert P.H.J. Schenning: 0000-0002-3485-1984

Notes

The authors declare no competing financial interest.

\section{ACKNOWLEDGMENTS}

The authors thank Rob Verpaalen and Marina Pilz Da Cunha for the XRD measurements and Dr. Alberto Belmonte Parra, Dr. Dirk Jan Mulder, and Sterre Bakker for helpful discussions. We are grateful for the financial support by The Netherlands Organization for Scientific Research (TOP-PUNT 718.016.003).

\section{REFERENCES}

(1) Vennes, M.; Martin, S.; Gisler, T.; Zentel, R. Anisotropic Particles from LC Polymers for Optical Manipulation. Macromolecules 2006, 39 (24), 8326-8333.

(2) Haseloh, S.; Zentel, R. Synthesis of Liquid-Crystalline Colloids in Nonpolar Media and their Manipulation in Electric Fields. Macromol. Chem. Phys. 2009, 210 (17), 1394-1401.

(3) Beltran-Gracia, E.; Parri, O. L. A new twist on cholesteric films by using reactive mesogen particles. J. Mater. Chem. C 2015, 3 (43), 11335-11340.

(4) Belmonte, A.; Bus, T.; Broer, D. J.; Schenning, A. Patterned FullColor Reflective Coatings Based on Photonic Cholesteric LiquidCrystalline Particles. ACS Appl. Mater. Interfaces 2019, 11 (15), 14376-14382.

(5) Humar, M.; Muševič, I. 3D microlasers from self-assembled cholesteric liquid-crystal microdroplets. Opt. Express 2010, 18 (26), 26995-27003.
(6) van Kuringen, H. P. C.; Mulder, D. J.; Beltran, E.; Broer, D. J.; Schenning, A. P. H. J. Nanoporous polymer particles made by suspension polymerization: spontaneous symmetry breaking in hydrogen bonded smectic liquid crystalline droplets and high adsorption characteristics. Polym. Chem. 2016, 7 (29), 4712-4716.

(7) Vennes, M.; Zentel, R.; Rossle, M.; Stepputat, M.; Kolb, U. Smectic Liquid-Crystalline Colloids by Miniemulsion Techniques. Adv. Mater. 2005, 17, 2123-2127.

(8) Mehravar, E.; Iturrospe, A.; Arbe, A.; Asua, J. M.; Leiza, J. R. Phase behavior of side-chain liquid-crystalline polymers containing biphenyl mesogens with different spacer lengths synthesized via miniemulsion polymerization. Polym. Chem. 2016, 7 (29), 47364750.

(9) Haseloh, S.; Ohm, C.; Smallwood, F.; Zentel, R. Nanosized shape-changing colloids from liquid crystalline elastomers. Macromol. Rapid Commun. 2011, 32 (1), 88-93.

(10) Wang, X.; Bukusoglu, E.; Abbott, N. L. A Practical Guide to the Preparation of Liquid Crystal-Templated Microparticles. Chem. Mater. 2017, 29 (1), 53-61.

(11) Ohm, C.; Serra, C.; Zentel, R. A continuous flow synthesis of micrometer-sized actuators from liquid crystalline elastomers. $A d v$. Mater. 2009, 21 (47), 4859-62.

(12) Lee, S. S.; Seo, H. J.; Kim, Y. H.; Kim, S. H. Structural Color Palettes of Core-Shell Photonic Ink Capsules Containing Cholesteric Liquid Crystals. Adv. Mater. 2017, 29 (23), 1606894.

(13) Fleischmann, E. K.; Liang, H. L.; Kapernaum, N.; Giesselmann, F.; Lagerwall, J.; Zentel, R. One-piece micropumps from liquid crystalline core-shell particles. Nat. Commun. 2012, 3, 1178.

(14) Li, Y.; Jun-Yan Suen, J.; Prince, E.; Larin, E. M.; Klinkova, A.; Therien-Aubin, H.; Zhu, S.; Yang, B.; Helmy, A. S.; Lavrentovich, O. D.; Kumacheva, E. Colloidal cholesteric liquid crystal in spherical confinement. Nat. Commun. 2016, 7, 12520.

(15) Hessberger, T.; Braun, L.; Zentel, R. Microfluidic Synthesis of Actuating Microparticles from a Thiol-Ene Based Main-Chain Liquid Crystalline Elastomer. Polymers 2016, 8 (12), 410.

(16) Vennes, M.; Zentel, R. Liquid-Crystalline Colloidal Particles. Macromol. Chem. Phys. 2004, 205 (17), 2303-2311.

(17) Haseloh, S.; van der Schoot, P.; Zentel, R. Control of mesogen configuration in colloids of liquid crystalline polymers. Soft Matter 2010, 6 (17), 4112.

(18) Song, J.-S.; Winnik, M. A. Cross-Linked, Monodisperse, Micron-Sized Polystyrene Particles by Two-Stage Dispersion Polymerization. Macromolecules 2005, 38 (20), 8300-8307.

(19) Song, J.-S.; Chagal, L.; Winnik, M. A. Monodisperse Micrometer-Size Carboxyl-Functionalized Polystyrene Particles Obtained by Two-Stage Dispersion Polymerization. Macromolecules 2006, 39 (17), 5729-5737.

(20) Downey, J. S.; Frank, R. S.; Li, W.-H.; Stöver, H. D. H. Growth Mechanism of Poly(divinylbenzene) Microspheres in Precipitation Polymerization. Macromolecules 1999, 32 (9), 2838-2844.

(21) Frank, R. S.; Downey, J. S.; Yu, K.; Stöver, H. D. H. Poly(divinylbenzene-alt-maleic anhydride) Microgels: Intermediates to Microspheres and Macrogels in Cross-Linking Copolymerization. Macromolecules 2002, 35 (7), 2728-2735.

(22) Zheng, G.; Stöver, H. D. H. Grafting of Poly(alkyl (meth)acrylates) from Swellable Poly(DVB80-co-HEMA) Microspheres by Atom Transfer Radical Polymerization. Macromolecules 2002, 35 (20), 7612-7619.

(23) Qi, D.; Yang, X.; Huang, W. Preparation of monodisperse fluorescent core-shell polymer microspheres with functional groups in the shell layer by two-stage distillation-precipitation polymerization. Polym. Int. 2007, 56 (2), 208-213.

(24) Li, W.-H.; Stöver, H. D. H. Monodisperse Cross-Linked CoreShell Polymer Microspheres by Precipitation Polymerization. Macromolecules 2000, 33 (12), 4354-4360.

(25) Li, G.; Yang, X.; Bai, F. A facile route to poly(divinylbenzene) hollow microspheres with pyridyl group on the interior surface. Polymer 2007, 48 (11), 3074-3081. 
(26) Li, G.; Yang, X.; Wang, B.; Wang, J.; Yang, X. Monodisperse temperature-responsive hollow polymer microspheres: Synthesis, characterization and biological application. Polymer 2008, 49 (16), 3436-3443.

(27) Yang, X.; Chen, L.; Huang, B.; Bai, F.; Yang, X. Synthesis of $\mathrm{pH}$-sensitive hollow polymer microspheres and their application as drug carriers. Polymer 2009, 50 (15), 3556-3563.

(28) Medina-Castillo, A. L.; Fernandez-Sanchez, J. F.; SeguraCarretero, A.; Fernandez-Gutierrez, A. Micrometer and Submicrometer Particles Prepared by Precipitation Polymerization: Thermodynamic Model and Experimental Evidence of the Relation between Flory's Parameter and Particle Size. Macromolecules 2010, 43 (13), 5804-5813.

(29) Spencer, W.; Sutter, J. R. Kinetic study of the monomer-dimer equilibrium of methylene blue in aqueous solution. J. Phys. Chem. 1979, 83 (12), 1573-1576.

(30) Braswell, E. Evidence for trimerization in aqueous solutions of methylene blue. J. Phys. Chem. 1968, 72 (7), 2477-2483.

(31) Golz, E. K.; Vander Griend, D. A. Modeling methylene blue aggregation in acidic solution to the limits of factor analysis. Anal. Chem. 2013, 85 (2), 1240-6.

(32) Ding, C.; Xu, S.; Wang, J.; Liu, Y.; Hu, X.; Chen, P.; Feng, S. Controlled loading and release of methylene blue from LbL polyurethane/poly(acrylic acid) film. Polym. Adv. Technol. 2012, 23 (9), 1283-1286.

(33) Deleers, M.; Servais, J.-P.; Wülfert, E. Micromolar concentrations of $\mathrm{Al} 3+$ induce phase separation, aggregation and dye release in phosphatidylserine-containing lipid vesicles. Biochim. Biophys. Acta, Biomembr. 1985, 813 (2), 195-200. 